\title{
An analysis of an activated sludge process containing a sludge disintegration system
}

\author{
$\underline{\text { M.I. Nelson }}^{\text {a }}$, E. Balakrishnan ${ }^{\text {b }}$

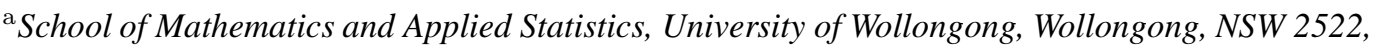 \\ Australia \\ ${ }^{\mathrm{b}}$ Department of Mathematics and Statistics, Sultan Qaboos University, Sultanate of Oman \\ Email: nelsonm@member.ams.org
}

\begin{abstract}
A continuous flow bioreactor is a well-stirred vessel containing microorganisms $(X)$ through which a substrate $(S)$ flows at a continuous rate. The microorganisms grow through the consumption of the substrate, producing more microorganisms and products. The products will typically contain carbon dioxide, nitrogen, water and other species, including biological compounds, specific to the process under consideration. The nature of these products is unimportant in this study. Unused substrate, microorganisms, and products flow out of the reactor. The use of a continuous flow bioreactor to treat sewage or industrial wastewaters is known as the activated sludge process.
\end{abstract}

One drawback associated with the activated sludge process is the production of 'sludge'. Traditional methods for disposing of excess sludge, which include incineration, the use of landfill sites and dumping at sea, are becoming increasingly regulated in many countries due to environmental concerns about the presence of potentially toxic elements in the sewage sludge. Furthermore, a combination of the limited amount of land available for landfill, particularly in urban areas, with stringent legislation has seen the economic costs of using landfill sites to increase sharply. It should be noted that incineration does not eliminate the need for landfill sites as a product of incineration is an ash containing high heavy materials content and general toxicity. Thus there is a pressing need, and growing interest, in methods that reduce the volume and mass of excess sludge produced as part of biological wastewater treatment processes.

A promising method to reduce excess sludge production is to increase the biodegradability of the sludge by disintegrating it within the reactor. This approach works primarily by causing the disintegration of bacterial cell walls. Among the many techniques that have been reported for application to the activated sludge process, chemical treatments and ozone treatments have been the most widely adopted commercially [Oh et al (2007)]. In processes involving ozonation a part of the sludge is removed from the reactor and treated with ozone in a sludge disintegrator. This ozonation stage converts the live sludge into a mixture of soluble substrate and particulates. The liquidized sludge is then returned to the bioreactor as a feed solution where the soluble substrate is biodegraded by live sludge. These techniques have shown to lead to much lower levels of MLSS (mixed liquor suspended solids).

A simple model is considered for a reactor cascade in which each reactor may be connected to both a settling unit and a sludge disintegration unit (SDU). The sludge disintegration unit is not modelled per se. Instead sludge disintegration terms are added to a conventional activated sludge model. These terms assume that the disintegrator unit destroys the biochemical activity of the sludge, converting a fraction, $\alpha$, directly into usable substrate and the remainder, $(1-\alpha)$, into organic particulates. We obtain a qualitative understanding of the performance of the process by finding the steady-state solutions of the model and determining their stability.

For a specified mixed liquor suspended solids (MLSS) content the values of the dimensionless residence time and the sludge disintegration factor are determined that ensure zero excess sludge production. We show that if the sludge disintegration factor is sufficiently high then the MLSS content is guaranteed to be below the target value provided that the residence time is higher than the washout value.

Keywords: activated sludge; CSTR; modelling; reactor cascade, sludge disintegration; sludge reduction; stirred tank; wastewater treatment. 


\section{INTRODUCTION}

The activated sludge process is the most widely used biological wastewater treatment method for domestic and industrial wastewaters [Wei et al (2003)]. One drawback associated with it is the production of 'sludge'. The expense for treating excess sludge can account for 50-60\% of the total operating costs in a wastewater treatment plant [Canales and Poles (1994); Egemen et al (2001); Nowack (2006)].

Methods that have been investigated to reduce excess sludge production include the addition of anoxic/anaerobic stages [Barker and Dold (1996); Yoon (2003)], enzyme treatment [Barjenbruch and Kopplow (2003)], freezing and thawing [Chi et al (1999)], an inclined-plate membrane bioreactor [Xing et al (2006)], ozonation [Yasui et al (1996); Sakai et al (1997); Egemen et al (2001); Song et al (2003); Wang et al (2008)], ozonation combined with chemical treatment [Oh et al (2007)], thermal treatment [Goma et al (1997); Kepp et al ( 1999)], and ultrasound [Yoon (2004)].

We consider a reactor cascade model for the activated sludge process in which each reactor may be connected to a settling unit and/or a sludge disintegration unit. This is an extension of an earlier model which investigated the operation of a membrane bioreactor connected to a sludge disintegration unit [Yoon (2003)]. We obtain a qualitative understanding of the relationship between operational parameters and the conditions for zero excess sludge production by finding the steady-state solutions of the model.

\section{EQUATIONS}

In this section we write down the model equations for the concentration of microorganisms, substrate and particulates for a reactor cascade in which each reactor is well-stirred and well aerated, possibly connected to a settling unit and a sludge disintegration unit. A reactor schematic is shown in figure 1.

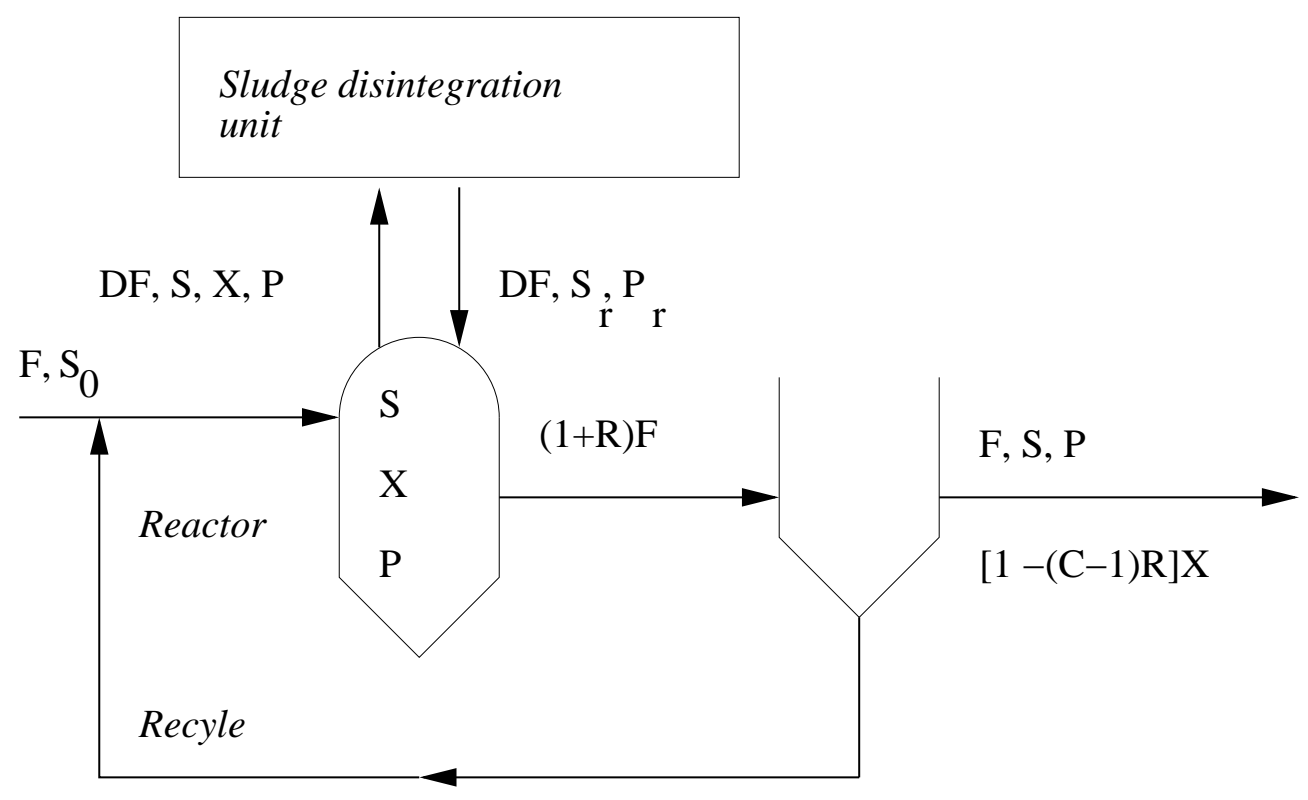

RF. S. CX. P

Figure 1. Schematic figure of a bioreactor with recycle and a SDU. The volume flux through the reactor is $F$, with $D F$ diverted into the SDU and $R F$ recycled through the reactor. The initial substrate concentration is $S_{0}$. The SDU returns substrate and product concentrations $S_{r}$ and $P_{r}$ to the reactor.

\subsection{Biochemical processes}

The biochemical processes occurring in the model are summarised as follows [Yoon (2003)] 
M.I. Nelson and E. Balakrishnan. An analysis of an activated sludge process...

Consumption of substrate $(S)$

$$
S \underset{X}{\stackrel{\mu(S)}{\longrightarrow}} Y \cdot X+\text { products }
$$

Death of biomass $(X)$ in the bioreactor

$$
X \stackrel{k_{d}}{\longrightarrow} \text { stuff }
$$

Hydrolysis of particulates $(P)$

$$
P \stackrel{k_{h}}{\longrightarrow} \beta S \text {. }
$$

Disintegration of biomass in the SDU

$$
X \stackrel{k=\infty}{\longrightarrow} \alpha \beta S+(1-\alpha) P .
$$

Disintegration of particulates in the SDU

$$
P \stackrel{k=\infty}{\longrightarrow} \alpha \beta S+(1-\alpha) P .
$$

In these equations $Y$ is a yield factor, $k_{d}$ is a decay coefficient, $k_{h}$ is a hydrolysis rate, $\alpha$ is the sludge solubilization efficiency, $\beta$ is a conversion efficiency and $\mu(S)$ is the specific growth rate model.

\subsection{The dimensional model}

Combining the biochemical processes from section 2.1 with the standard modelling paradigm for a wellstirred reactor cascade containing $n$ reactors we obtained the system of equations.

Concentration of substrate in reactor $i\left(S_{i}\right)$

$$
\frac{\mathrm{d} S_{i}}{\mathrm{~d} t}=\frac{n}{\tau_{t}} \cdot\left(S_{i-1}-S_{i}\right)+\frac{n}{\tau_{t}} \cdot D_{i}\left(S_{i . r}-S_{i}\right)+\beta k_{h} P_{i}-\frac{\mu_{m} S_{i}}{K_{S}+S_{i}} \cdot \frac{1}{Y} \cdot X_{i} .
$$

Concentration of biomass in reactor $i\left(X_{i}\right)$

$$
\left.\frac{\mathrm{d} X_{i}}{\mathrm{~d} t}=\frac{n}{\tau_{t}} \cdot\left(X_{i-1}-X_{i}\right)-\frac{n}{\tau_{t}} \cdot D_{i} X_{i}+\frac{\mu_{m} S_{i}}{K_{S}+S_{i}} \cdot X_{i}-k_{d} X_{i}+\frac{n}{\tau_{t}} \cdot R_{i}(\mathcal{C}\rangle-1\right) X_{i}
$$

Concentration of particulates in reactor $i\left(P_{i}\right)$

$$
\frac{\mathrm{d} P_{i}}{\mathrm{~d} t}=\frac{n}{\tau_{t}} \cdot\left(P_{i-1}-P_{i}\right)+\frac{n}{\tau_{t}} \cdot D_{i}\left(P_{i . r}-P_{i}\right)-k_{h} P_{i}
$$

Concentration of substrate leaving the sludge disintegration unit connected to reactor $i\left(S_{i . r}\right)$.

$$
S_{i . r}=S_{i}+\alpha \beta\left(X_{i}+P_{i}\right) .
$$

Concentration of damaged biomass leaving the sludge disintegration unit connected to reactor $i\left(P_{i . r}\right)$

$$
P_{i . r}=(1-\alpha)\left(X_{i}+P_{i}\right) .
$$

In equations (6)-(8) the primary bifurcation parameter is the total residence time $\left(\tau_{t}\right)$. The secondary bifurcation parameter is the sludge disintegration factor $\left(D_{i}\right)$. The definition of the parameters in the model, and their typical values, are provided by Yoon (2003). We note that $X_{0}=P_{0}=0$.

The quantity of particular interest in this system is the steady-state value for the mixed liquor suspended solids (MLSS). This is equivalent to the concentration of sludge within the reactor and is defined by

$$
\text { MLSS }=X+P .
$$

If possible the reactor should be operated to ensure that the MLSS is below a target value, MLSS target $_{\text {. }}$ The target value was taken to be $12,000 \mathrm{mg} \mathrm{L}^{-1}$ [Yoon (2003)]. 


\subsection{The dimensionless model}

By introducing dimensionless variables for the substrate concentration $\left[S^{*}=S / K_{s}\right]$, the cell mass concentration $\left[X^{*}=X /\left(Y K_{s}\right)\right]$, the particulate concentration $\left[P^{*}=P /\left(Y K_{s}\right)\right]$, and time $\left[t^{*}=\mu_{m} t\right]$ the dimensional model, equations (6)-(8), can be written in the dimensionless form

$$
\begin{aligned}
\frac{\mathrm{d} S_{i}^{*}}{\mathrm{~d} t^{*}} & =\frac{n}{\tau_{t}^{*}}\left(S_{i-1}^{*}-S_{i}^{*}\right)+\frac{n}{\tau_{t}^{*}} \cdot \alpha \beta^{*} D_{i}\left(X_{i}^{*}+P_{i}^{*}\right)+\beta^{*} k_{h}^{*} P_{i}^{*}-\frac{S_{i}^{*}}{1+S_{i}^{*}} X_{i}^{*}, \\
\frac{\mathrm{d} X_{i}^{*}}{\mathrm{~d} t^{*}} & =\frac{n}{\tau_{t}^{*}}\left(X_{i-1}^{*}-X_{i}^{*}\right)+\left[\frac{n}{\tau_{t}^{*}} \cdot\left(R_{i}^{*}-D_{i}\right)+\frac{S_{i}^{*}}{1+S_{i}^{*}}-k_{d}^{*}\right] X_{i}^{*}, \\
\frac{\mathrm{d} P_{i}^{*}}{\mathrm{~d} t^{*}} & =\frac{n}{\tau_{t}^{*}}\left(P_{i-1}^{*}-P_{i}^{*}\right)+\frac{n D_{i}}{\tau_{t}^{*}}\left[(1-\alpha) X_{i}^{*}-\alpha P_{i}^{*}\right]-k_{h}^{*} P_{i}^{*} .
\end{aligned}
$$

Typical values for the dimensionless parameters are [Yoon (2003)]: $S_{0}^{*}=300, k_{d}^{*}=0.028, k_{h}^{*}=0.3$, $\beta^{*}=0.6$. The effective recycle parameter is bounded by $0 \leq R_{i}^{*} \leq 1$. We note that $X_{0}^{*}=P_{0}^{*}=0$.

The scaled value of the mixed liquor suspended solids (MLSS*) is given by

$$
\operatorname{MLSS}^{*}=X^{*}+P^{*} \text {. }
$$

The scaled target value us

$$
\mathrm{MLSS}_{\text {target }}^{*}=240 \text {. }
$$

\section{RESULTS}

\subsection{Steady-state solution branches}

There may be up to three solution branches: global washout, partial washout and no-washout. Along the global washout solution branch the steady-state solution in each reactor of the cascade is given by

$$
\left(S_{i}^{*}, X_{i}^{*}, P_{i}^{*}\right)=\left(S_{0}^{*}, 0,0\right), \quad i=1,2 \ldots n .
$$

This solution corresponds to process failure.

For the partial washout solution there is a critical reactor index $(j)$. Washout occurs in each reactor along the cascade before reactor $j$, i.e. for $1 \leq i \leq j-1$

$$
\left(S_{i}^{*}, X_{i}^{*}, P_{i}^{*}\right)=\left(S_{0}^{*}, 0,0\right) .
$$

In reactor $j$ there is a positive value for the steady-state concentrations of the substrate and microorganisms: $S_{j}^{*}>0 \& X_{j}^{*}>0$. The steady-state particulate concentration is non-negative: $P_{j}^{*} \geq 0$. The steady-state values in each subsequent reactor are positive.

The no-washout solution branch corresponds to a solution in which there are positive steady-state solutions in each reactor of the cascade. If the values for both $D_{i}^{*}$ and $R_{i}^{*}$ are identical throughout the reactor cascade, i.e. $D_{i}^{*}=D^{*}$ and $R_{i}^{*}=R^{*}$, then only the global washout solution and the no-washout solution branches exist.

\subsection{Stability of the steady-state solutions}

The global washout branch is always stable if

$$
k_{d}^{*}>\frac{S_{0}^{*}}{1+S_{0}^{*}} .
$$

It is stable when equality holds provided that $R_{i}^{*}-D_{i}^{*}-1 \neq 0$.

If $k_{d}^{*}<\frac{S_{0}^{*}}{1+S_{0}^{*}}$ then the global washout steady-state is stable provided

$$
\tau_{t}^{*}<\left[\frac{n\left(1+S_{0}^{*}\right)}{S_{0}^{*}-\left(1+S_{0}^{*}\right) k_{d}^{*}}\right] \cdot \min \left(1+D_{i}^{*}-R_{i}^{*}\right) .
$$


If the partial washout solution branch exists then it is stable provided that

$$
\left[\frac{n\left(1+S_{0}^{*}\right)}{S_{0}^{*}-\left(1+S_{0}^{*}\right) k_{d}^{*}}\right] \cdot \min \left(1+D_{i}^{*}-R_{i}^{*}\right) \cdot<\tau^{*}<\left[\frac{n\left(1+S_{0}^{*}\right)}{S_{0}^{*}-\left(1+S_{0}^{*}\right) k_{d}^{*}}\right] \cdot \max \left(1+D_{i}^{*}-R_{i}^{*}\right) .
$$

The no-washout solution branch is stable when

$$
\tau_{t}^{*}>\left[\frac{n\left(1+S_{0}^{*}\right)}{S_{0}^{*}-\left(1+S_{0}^{*}\right) k_{d}^{*}}\right] \cdot \max \left(1+D_{i}^{*}-R_{i}^{*}\right) .
$$

\subsection{Steady-state diagrams}

Figure 2 (a) shows the effluent concentration leaving both a single reactor and a double reactor cascade. The parameter values have been chosen so that neither a settling unit nor a sludge disintegration unit (SDU) are employed. In the single reactor the global washout steady-state branch is stable for $\tau_{t}^{*} \leq 1.032$. In the cascade the global washout steady-state branch is stable for $\tau_{t}^{*} \leq 2.064$. There is a small range of values for the residence time over which the effluent concentration leaving a single reactor is lower than leaving a cascade. However, for moderate values of the residence time the effluent concentration leaving the cascade is several orders of magnitude lower than leaving the single reactor; this is one reason why cascades are used to treat slurries and wastewaters.

Figure 2 (b) shows the MLSS leaving both a single reactor and a double reactor cascade. The absence of a SDU $\left(D_{1}^{*}=D_{2}^{*}=0\right)$ means that particulates are not formed in the cascade; the MLSS concentration is equal to the concentration of micro-organisms. For moderate values of the residence time there is little difference in performance between the systems. For larger values, the MLSS leaving the cascade is slightly lower. The target MLSS line intersects the steady-state MLSS curve at two locations. Only the higher of the two corresponding residence times is of interest. For the single reactor and the cascade the target MLSS is achieved when $\tau_{t}^{*}=8.9$ and $\tau^{*}=8.4$ respectively.
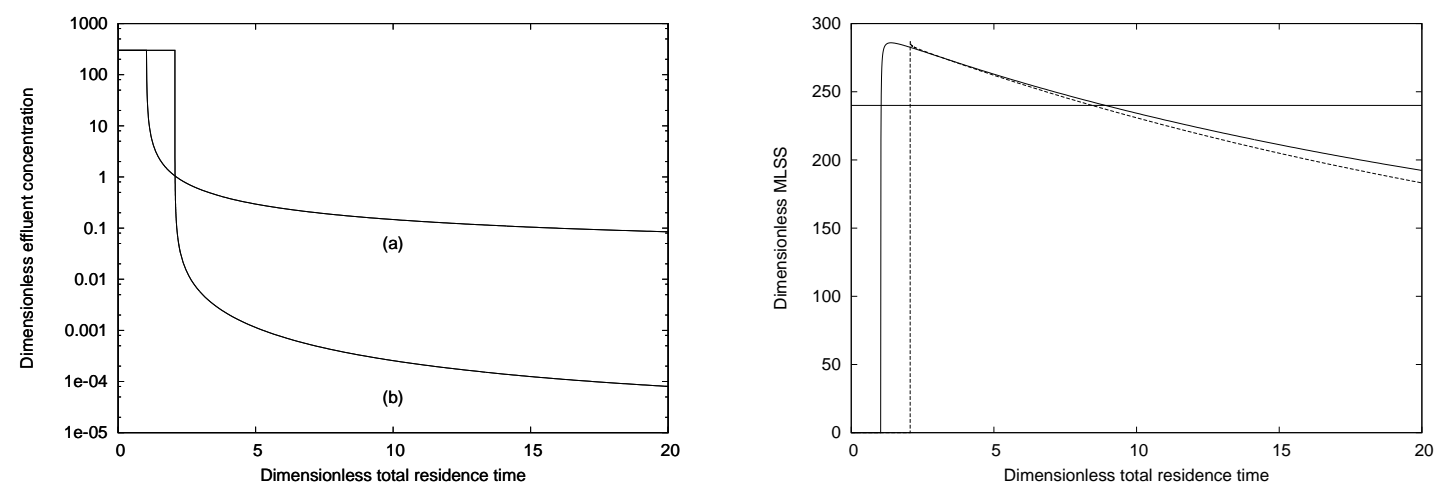

Figure 2. Steady-state diagrams showing the variation of the effluent concentration $\left(S_{e}^{*}\right)$ and MLSS as a function of the total dimensionless residence time $\left(\tau^{*}\right)$ in a single reactor (line a) and a double reactor cascade (line b). Parameter values: $D_{1}^{*}=D_{2}^{*}=0 ; R_{1}^{*}=R_{2}^{*}=0$.

Figure 3 shows steady-state diagrams for three double reactor cascades. In each configuration there are no settling units. Line (a) corresponds to the no-SDU case seen in figure 2. Lines (b) \& (c) represent cases when a SDU is placed around the first and second reactor respectively.

Figure 3 (a) shows the effluent concentration as a function of the total residence time in the cascade. disintegration unit (SDU) are employed. In configuration (b) the global washout steady-state branch is stable for $\tau^{*} \leq 2.06$, the partial washout steady-state branch is stable for $2.06 \leq \tau^{*} \leq 3.10$ and the no-washout branch is stable for $\tau^{*}>3.10$. In configuration (c) the global washout branch is stable for $\tau^{*} \leq 2.06$. 
The operation of a SDU around either reactor (1) or reactor (2) increases the effluent concentration leaving the reactor. This reflects the fact that the SDU increases the food supply whilst decreasing the amount of live biomass. There is a small operating region in which configuration (c) has a lower effluent concentration than configuration (b), but at moderate values of the total residence time the configuration with the SDU operating around the first reactor has the lower effluent concentration. At these residence times the effluent concentration from Reactor configuration (c) is still lower than that from a single reactor.

Figure 2 (b) shows the MLSS leaving the three reactor double reactor configurations. Although particulates are produced by the action of the SDU, the MLSS is generally lower than the configuration without a SDU. There is a small parameter region in which the MLSS value is higher in configuration (b) than in configuration (a): in this region the operation of a SDU around the first reactor causes the system to stabilise around the partial washout branch.

For moderate values of the residence time there is little difference in performance between systems (b) and (c). The target MLSS line intersects the steady-state MLSS curve at $\tau^{*}=3.13$ for configuration (b). This is a considerable decrease in the value compared to configuration (a), though the penalty for this is at there is a higher effluent concentration. For configuration (c) the maximum MLSS is below the target level: thus this configuration operates in a state of 'negative excess sludge' regardless of the residence time.
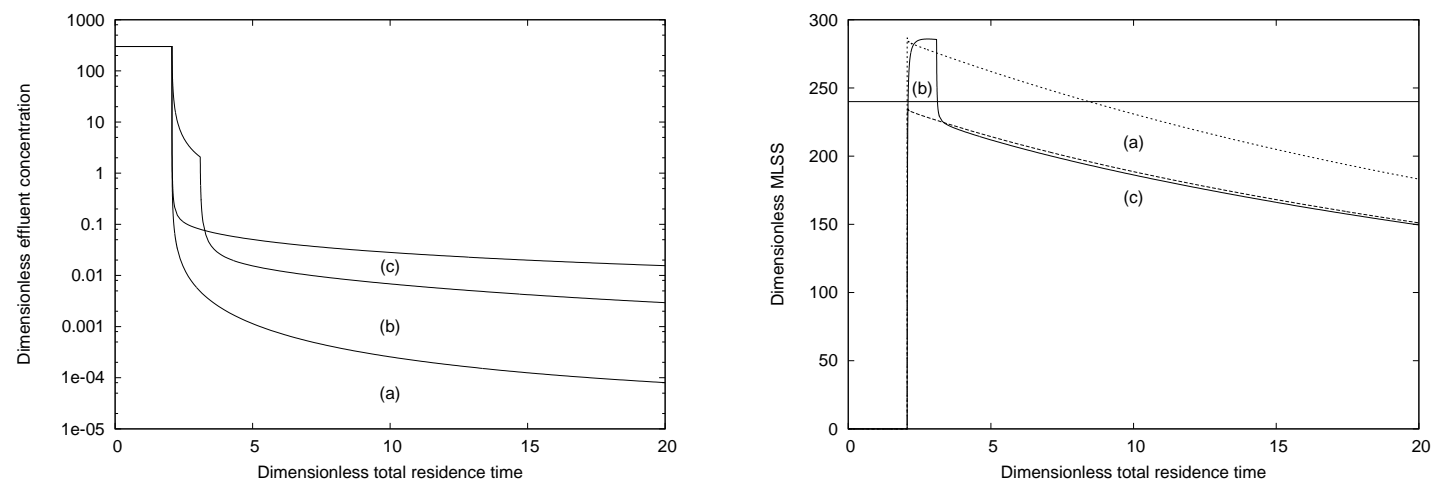

Figure 3. Steady-state diagrams showing the variation of the effluent concentration $\left(S_{e}^{*}\right)$ and MLSS as a function of the total dimensionless residence time $\left(\tau^{*}\right)$. in a double reactor cascade without any settling units. Parameter values: (a) no SDU; (b) $D_{1}^{*}=0.5$; (c) $D_{2}^{*}=0.5$.

\section{CONCLUSIONS}

We have investigated a model for the activated sludge process connected to a sludge disintegration system. The biochemical model used was originally proposed by Yoon (2003). It is assumed that the disintegrator unit is $100 \%$ efficient. Consequently the disintegrator unit is modelled by two algebraic equations.

We have used steady-state methods to analyse the behaviour of the activated sludge model. There may be three solution branches corresponding to states of global washout, partial washout and no-washout. In the partial washout solution reactors at the 'start' of the cascade are in a state of washout whilst those at the 'end' of the cascade are in a state of no-washout. This solution branch is formed when a SDU is operated around the 'start' of the reactor cascade. The stability of the solution branches has been indicated.

The operation of a SDU increases the effluent concentration leaving a cascade (which is bad) whilst reducing the MLSS concentration, or 'sludge', leaving the cascade (which is good). Furthermore the SDU unit decreases the residence time required to achieve a target MLSS concentration. We have shown that it is possible to ensure that a reactor-cascade always operates below the target value.

Investigations are currently on going to analyse this system in a more systematic manner. 
M.I. Nelson and E. Balakrishnan. An analysis of an activated sludge process...

\section{ACKNOWLEDGEMENT}

This work is supported by a grant from Sultan Qaboos University.

\section{REFERENCES}

M. Barjenbruch and O. Kopplow. (2003). Enzymatic, mechanical and thermal pre-treatment of surplus sludge. Advances in Environmental Research 7(3), 715-720.

P.S. Barker and P.L. Dold. (1996). Sludge production and oxygen demand in nutrient removal activated sludge systems. Water Science and Technology 34(5), 43-50.

A.P. Canales and J.L. Poles. (1994). Decreased sludge production strategy for domestic wastewater treatment. Water Science and Technology 30(8), 97-106.

C.P. Chu, W.H. Feng, B.V. Chang, C.H. Chou, and D.J. Lee. (1999). Reduction in microbial density level through freezing and thawing. Water Research 33, 3532-3535.

E. Egemen, J. Corpening, and N. Nirmalakhandan. (2001). Evaluation of an ozonation system for reduced waste sludge generation. Water Science \& Technology 44(2-3), 445-52.

G. Goma, J.L. Rols, and C. Pareilleux. (1997). Membrane bioreactor for wastewater treatment: reduction of sludge production. In D.L. Wise, editor, Global Environment Biotechnology, pages 461-467, Boston, 1997. Kluwer Academic Publishers.

U. Kepp, L. Machenbach, N. Weisz, and O.E. Solheim. (1999). Enhanced stabilisation of sewage sludge through thermal hydrolysis - three years of experience with a full scale plant. Water Science and Technology 42(9), 89-96.

O. Nowak. (2006). Optimizing the use of sludge treatment facilities at municipal WWTPs. Journal of Environmental Science and Health Part A - Toxic/Hazardous Substances \& Environmental Engineering 41(9), 1807-1817.

Y-K. Oh, K-R. Lee, K-B. Ko, and I-T Yeom. (2007). Effects of chemical sludge disintegration on the performance of wastewater treatment by membrane bioreactor. Water Research 41, 2665-2671.

Y. Sakai, T. Fukase, and H. Yasui. (1997). An activated sludge process without excess sludge production. Water Science and Technology 36(11), 163-170.

K. Song, Y. Chung, K. Ahn, J. Cho, and H. Yun. (2003). Performance of membrane bioreactor system with sludge ozonation process for minimization of excess sludge production. Desalination 157, 353359.

Z. Wang, L. Wang, B.Z. Wang, Y.F. Jiang, and S. Liu. (2008). Bench-scale study on zero excess activated sludge production process coupled with ozonation unit in membrane bioreactor. Journal of Environmental Science and Health, Part A 43(11), 1325-1332.

Y. Wei, Van Houten R.T. A.R. Borger, D.H. Eikelboom, and Y. Fan. (2003). Minimization of excess sludge production for biological wastewater treatment. Water Research 37, 4553-4467.

C-H. Xing, K. Yamamoto, and K. Fukushi. (2006). Performance of an inclined-plate membrane bioreactor at zero excess sludge discharge. Journal of Membrane Science 275, 175-186.

H. Yasui, K. Nakamura, S. Sakuma, M. Iwasaki, and Y. Sakai. (1996). A full-scale operation of a novel activated sludge process without excess sludge production. Water Science and Technology 34(3-4), 395-404.

S-H. Yoon. (2003). Important operational parameters of membrane bioreactor-sludge disintegration (MBR-SD) system for zero excess sludge production. Water Research 37, 1921-1931.

S-H. Yoon, H-S. Kim, and S. Lee. (2004). Incorporation of ultrasonic cell disintegration into a membrane bioreactor for zero sludge production. Process Biochemistry 39(12), 1923-1929. 